\title{
INSTABILITY OF A SURFACE ELASTIC LINE ON AN UNNATURAL RAMP
}

\author{
BY \\ GERALD S. MANNING \\ Rutgers University, New Brunswick, New Jersey
}

\begin{abstract}
A line with bending resilience (Hooke's Law constant $b$ ) but invariant length $l$ (elastic line) is confined to a cylindrical surface of radius $R$. A clamp at its starting point requires its initial direction always to lie along the base circle, but its end point and direction are not constrained. The trajectory in which the elastic line lies for its entire length along the circle is then an equilibrium path. Since the elastic line can straighten by swerving toward the generators of the cylinder, however, the circumferential trajectory is unstable if $l / R$ exceeds the critical value $\pi / 2^{3 / 2}$ $(\approx 1.1107)$. To improve stability, we have constructed a ramp along the circle in the form of a potential energy trough (depth $\gamma$, width $\delta$ ), which, by means of surface forces, acts to attract the elastic line toward the circle. When the ramp strength is built up to a level $\gamma \delta R^{4} / b \approx 5.1743$, the stability of the circumferential trajectory is somewhat improved, lengths in excess of $l / R \approx 1.4178$ now being unstable. But if the ramp strength is increased above this critical value, even if only slightly, the circumferential trajectory for arbitrarily long elastic lines abruptly becomes stable.
\end{abstract}

1. Introduction. An elastic line of invariant length $l$ may be defined by its stress energy $K$,

$$
K=\frac{1}{2} b \int_{0}^{l} \kappa^{2} d s
$$

with $b$ the Hooke's Law constant and $\kappa^{2}(s)$ the square curvature at arc length $s$ along the line $[1,2,3]$. The structure of the biological assembly known as the nucleosome [4] raises the question of the equilibrium trajectories and their stabilities of an elastic line (model for DNA) confined to a curved surface (model for exterior of protein core). Conventional Euler-Lagrange equations for $K$ with trajectories on a surface were developed in [1] with the point and tangent at $s=0$ specified. Natural boundary conditions at $s=l$ were employed; that is, aside from the constaints at the starting point, the elastic line was allowed to search freely anywhere on the surface for its equilibria. (Physically, the elastic line is held to the surface by a strong enough, uniform attractive potential over the entire surface. Different trajectories are then energetically distinguishable only through different values of the bending

Received July 24, 1989.

Research partially supported by NIH Grant GM36284.

(C)1990 Brown University 
energy $K$ on the surface. The surface forces brought into the problem below give a surface-varying potential energy on top of the uniform attraction.)

The equilibrium trajectory lies always along a geodesic curve if and only if the surface is a plane or sphere $[1,3]$. On a cylinder, which is thought to model the nucleosome best, the equilibrium trajectory is geodesic if the starting direction is axial (then the equilibrium is along a generator of the cylinder). But if the starting direction is oblique, the equilibrium trajectory deviates from the helix (geodesic) with coincident starting direction in such a way that the pitch continuously increases [2]. The oblique elastic line at equilibrium thus winds around the cylinder, but at a much slower rate than the helix. For asymptotically large $s$, the winding number of the elastic line increases, not in proportion to $s$, nor even to any fractional power of $s$, but logarithmically.

The tendency of an elastic line to straighten toward the axial direction on a cylinder assumes its most striking form when the starting direction is orthogonal to the generators, along a base circle [2]. The elastic line remains geodesic along the circle if it is not too long, specifically if $l / R<\pi / 2^{3 / 2}$, where $R$ is the radius of the circle. An elastic line of this critical length lying along the circle is in neutral equilibrium. A longer line is unstable in the circumferential trajectory, exchanging stability for a path with an axial component.

The intrinsic equations for an equilibrium elastic line on a surface provide a more elegant formulation that avoids use of Lagrange multipliers [3]. They are readily generalized to the case of an elastic line with equilibrium trajectories determined not only by surface curvature but also by applied forces directed tangentially to the surface (surface forces) [5]. In this paper we use surface forces to build a "ramp" along the base circle of a cylinder in an effort to improve the stability of an elastic line in its equilibrium path along the circle. Biophysical motivation is deferred to the last section. We begin with a review of the intrinsic equations in the ramp-free case.

2. Intrinsic equations in the absence of a ramp [3]. If the elastic line is not subjected to surface forces, its equilibrium trajectories on an oriented surface satisfy the intrinsic differential equation,

$$
B\left[\kappa_{n}, \kappa_{g}, \tau_{g}\right]=0
$$

where the "bending operator" $B$ acts on the normal curvature $\kappa_{n}(s)$ of the trajectory, its geodesic curvature $\kappa_{g}(s)$, and its geodesic torsion $\tau_{g}(s)$ to give (primes denote derivatives with respect to $S$ ),

$$
\begin{aligned}
(2 / b) B\left[\kappa_{n}, \kappa_{g}, \tau_{g}\right]= & 2 \kappa_{g}^{\prime \prime}-4 \tau_{g} \kappa_{n}^{\prime}-2 \kappa_{n} \tau_{g}^{\prime} \\
& +\kappa_{g}\left[\kappa^{2}-2 \tau_{g}^{2}+\kappa^{2}(l)\right] .
\end{aligned}
$$

Just as the curvature $\kappa(s)$ and torsion of a space curve provide an intrinsic description of the curve free from reference to the enveloping space, the functions $\kappa_{n}, \kappa_{g}$, and $\tau_{g}$ are intrinsic to a curve contained in a surface. The normal and geodesic curvatures combine to yield the full curvature,

$$
\kappa^{2}=\kappa_{n}^{2}+\kappa_{g}^{2}
$$


The quantity $\kappa(l)$ in Eq. (3) is the value of the curvature $\kappa(s)$ at the end point of the elastic line $s=l$. It is a parameter that must generally be determined as part of the solution of the problem.

The geometric meaning of the two curvature components and the geodesic torsion are contained in equations analogous to the Frenet-Sorret formulas,

$$
\left(\begin{array}{c}
\mathbf{T}^{\prime} \\
\mathbf{Q}^{\prime} \\
\mathbf{N}^{\prime}
\end{array}\right)=\left(\begin{array}{ccc}
0 & \kappa_{g} & \kappa_{n} \\
-\kappa_{g} & 0 & \tau_{g} \\
-\kappa_{n} & -\tau_{g} & 0
\end{array}\right)\left(\begin{array}{l}
\mathbf{T} \\
\mathbf{Q} \\
\mathbf{N}
\end{array}\right) .
$$

The unit vectors $\mathbf{T}(s)$ and $\mathbf{Q}(s)$ are, respectively, the tangent to the trajectory in the direction of increasing $s$ and a surface tangent orthogonal to $\mathbf{T}$, oriented consistently with surface coordinates $(u, v)$ at $s$. The unit surface normal $\mathbf{N}(s)$ equals $\mathbf{T} \times \mathbf{Q}$.

Two boundary conditions at the starting point $s=0$ are specified, the values of the surface coordinates $(u, v)$ and the tangent $\mathbf{T}$ there. In other words, we have the starting location and direction. Two more boundary conditions arise from the natural (free) movement of the end point $s=l$,

$$
\begin{aligned}
\kappa_{g}(l) & =0 \\
\kappa_{g}^{\prime}(l) & =2 \kappa_{n}(l) \tau_{g}(l) .
\end{aligned}
$$

Eq. (6) implies that the term $\kappa^{2}(l)$ in Eq. (3) may be replaced by $\kappa_{n}^{2}(l)$.

3. An unnatural ramp. If forces tangent to the surface are imposed on the elastic line, the trajectory at equilibrium will be constrained, or, as we may say, unnatural. Let the energy due to surface forces of the elastic line in a given trajectory $[u(s), v(s)]$, be given by,

$$
W=-\gamma \int_{0}^{l} w[u(s), v(s)] d s
$$

where $\gamma$ is a constant that measures the strength of the ramp (i.e., the depth of the potential energy trough along the ramp) and $w(u, v)$ gives its shape. A segment $d s$ of the elastic line at arc length $s$ along the trajectory experiences a force tangential to the surface characterized by the surface gradient of the potential energy $-\gamma w d s$.

The equilibrium trajectories are extrema of the sum $K+W$ of stress and potential energies. The intrinsic equation in the presence of the ramp is [5],

$$
B\left[\kappa_{n}, \kappa_{g}, \tau_{g}\right]+E[w]=0
$$

where the "ramp operator" $E$ acts on $w$ along the trajectory to give,

$$
-(1 / \gamma) E[w]=\partial_{\mathbf{Q}} w(s)-\kappa_{g}[w(s)-w(l)]
$$

$\partial_{\mathbf{Q}}$ denoting differentiation in the direction of surface tangent $\mathbf{Q}$ (orthogonal to the trajectory), and $w(l)$ the value of $w(s)$ at $s=l$, a parameter generally determined as part of the solution of the problem. The boundary conditions at $s=0, l$ are the same as for the ramp-free case.

We can get an immediate result for an elastic line on a cylinder directed at $s=0$ along the base circle, assuming that the ramp runs along the circle. The circle is 
a geodesic, so its geodesic curvature vanishes. It is a plane curve as well, so its geodesic torsion vanishes also. Hence, the trajectory lying entirely along the circle satisfies $B\left[\kappa_{n}, \kappa_{g}, \tau_{g}\right]=0$. The bottom of the potential energy trough runs along the circle, so the orthogonal derivative and (both factors of) the second term in brackets in Eq. (10) vanish. Thus $E[w]$ vanishes for the circumferential trajectory, and Eq. (9) is satisfied. Both boundary conditions, Eqs. (6) and (7), are obeyed. The elastic line running along the base circle for its entire length $l$ is in mechanical equilibrium.

4. Nearly circumferential trajectories. To investigate the stability of an elastic line in its circumferential trajectory, we want to consider trajectories that lie near it. Their geodesic curvatures and geodesic torsions are both small, and products in $B\left[\kappa_{n}, \kappa_{g}, \tau_{g}\right]$ involving as factors two or more of these functions or their derivatives may be neglected. Their normal curvatures are close to the constant curvature of the circle, so $\kappa_{n}^{\prime}$ is small for them also. Thus, for these near-lying trajectories,

$$
(2 / b) B\left[\kappa_{n}, \kappa_{g}, \tau_{g}\right]=2 \kappa_{g}^{\prime \prime}-2 \kappa_{n} \tau_{g}^{\prime}+\kappa_{g}\left[\kappa_{n}^{2}+\kappa_{n}^{2}(l)\right]
$$

Further simplification is achieved through use of the obvious coordinates for a cylinder, circumferential length $u$ (along the base circle) and axial length $v$ (along a generator). If $\theta(s)$ is the angle measured from the $u$-coordinate curve to the tangent $\mathbf{T}$ of the elastic line, both with origin on the elastic line at $s$, then we have the formulas (for any trajectory on a cylinder of radius $R$ [3]),

$$
\kappa_{g}=\theta^{\prime}, \quad \kappa_{n}=-R^{-1} \cos ^{2} \theta, \quad \tau_{g}=R^{-1} \cos \theta \sin \theta .
$$

The circumferential trajectory is characterized by the vanishing of $\theta$ along its entire length, and near-lying trajectories therefore have small values of $\theta$ (and its derivatives). With neglect of higher-order terms in $\theta$, then, we get,

$$
B\left[\kappa_{n}, \kappa_{g}, \tau_{g}\right]=b\left(\theta^{\prime \prime \prime}+2 R^{-2} \theta^{\prime}\right) .
$$

(It may also be checked directly from Eq. (12) that the terms neglected in passing from Eq. (3) to Eq. (11) are indeed higher-order in $\theta$.)

Turning to $E[w]$ in Eq. (10), we have the smallness of $\kappa_{g}$ for trajectories lying near the circle. Further, $w(s)-w(l)$ vanishes for the circumferential trajectory, which runs along the flat bottom of the potential energy ramp; therefore, $w(s)-w(l)$ is a small quantity for trajectories lying near the circle. The product $\kappa_{g}[w(s)-w(l)]$ is thus higher-order in smallness and may be neglected. (If our argument appears "soft," one may check that this term is actually third-order in $\theta$ and its derivatives from Eq. (12) and the following formulas for $w$.) $E[w]$ reduces to its first term $-\gamma \partial_{\mathbf{Q}} w$.

With surface coordinates $(u, v), u$ circumferential and $v$ axial, we may choose a truncated expansion for $u$,

$$
w(v)=1-\frac{1}{2} \delta v^{2}
$$

since trajectories close to the base circle, $v=0$, do not sense the presence of the higher-order terms. The constant $\delta$ controls the width of the ramp. The statement 
$\partial w / \partial u=0$ is the mathematical definition of what we mean by a ramp along the base circle. We can make the calculation

$$
\begin{aligned}
\partial_{\mathbf{Q}} w(s) & =-(\partial w / \partial u) \sin \theta+(\partial w / \partial v) \cos \theta \\
& =-\delta v \cos \theta \\
& =-\delta \cos \theta \int_{0}^{s} \sin \theta d s \\
& =-\delta \int_{0}^{s} \theta d s
\end{aligned}
$$

where the last line neglects terms higher-order in $\theta$. The conclusion is that,

$$
E[w]=\gamma \delta \int_{0}^{s} \theta d s
$$

for trajectories near the circle.

We have thus reduced the intrinsic equation, Eq. (9), for our nearly circumferential trajectories to

$$
b\left(\theta^{\prime \prime \prime}+2 R^{-2} \theta^{\prime}\right)+\gamma \delta \int_{0}^{s} \theta d s=0 .
$$

The first end-point condition, Eq. (6), becomes, from Eq. (12),

$$
\theta^{\prime}(l)=0
$$

while the second end-point condition, Eq. (7), reduces for small $\theta$ to,

$$
\theta^{\prime \prime}(l)+2 R^{-2} \theta(l)=0 .
$$

It is perhaps more convenient to work with dimensionless quantities. Let $\sigma=s / l$, and redefine "prime" to mean differentiation with respect to $\sigma$. Then,

$$
\theta^{\prime \prime \prime}+\alpha^{2} \theta^{\prime}+\beta^{2} \int_{0}^{\sigma} \theta d \sigma=0
$$

where

$$
\alpha=\sqrt{2} l / R, \quad \beta^{2}=\gamma \delta l^{4} / b
$$

are both dimensionless constants; and the two conditions at the end point $\sigma=1$ are,

$$
\theta^{\prime}(1)=0, \quad \theta^{\prime \prime}(1)+\alpha^{2} \theta(1)=0 .
$$

Finally, we introduce the function

$$
y(\sigma)=\int_{0}^{\sigma} \theta(\sigma) d \sigma
$$

in terms of which Eq. (19) becomes,

$$
y^{(\mathrm{iv})}+\alpha^{2} y^{\prime \prime}+\beta^{2} y=0
$$

a linear fourth-order equation with constant coefficients. There are two starting-point conditions,

$$
y(0)=y^{\prime}(0)=0
$$


the first of which follows from the definition of $y(\sigma)$, while the second states the clamped starting-point direction $\theta(0)=0$ along the base circle. The two end-point conditions become

$$
y^{\prime \prime}(1)=0, \quad y^{\prime \prime \prime}(1)+\alpha^{2} y^{\prime}(1)=0 .
$$

Identically vanishing $y$ (which implies the circumferential trajectory $\theta=0$, since $\theta=y^{\prime}$ ) is a solution of Eq. (23) with boundary conditions Eqs. (24) and (25). Are there nonvanishing solutions, and, if so, in what conditions do they emerge?

5. Stability analysis for the circumferential trajectory. A result valid for any surface and a sufficiently short elastic line with fixed starting-location and direction is that the geodesic trajectory provides the global energy minimum [1]. A sufficiently short elastic line has least energy as a geodesic arc. We proved this statement for the rampfree case, but installation of a ramp along the geodesic can only improve the stability (a competing trajectory not only has higher bending energy, but its ramp energy must be higher also if the ramp runs along the geodesic). So a sufficiently short elastic line running along the ramp-endowed base circle of a cylinder is strongly stable, in the sense that any other trajectory for it has higher energy.

The equilibrium trajectories for an elastic line of general length satisfy Eq. (23), which has as general solution,

$$
y(\sigma)=\sum_{m=1}^{4} A_{m} e^{a_{m} \sigma}
$$

where the four roots of the characteristic polynomial are the four combinations of the expression,

$$
\pm(\alpha / \sqrt{2}) \sqrt{-1 \pm \sqrt{1-p^{2}}}
$$

with the parameter $p^{2}$ defined by,

$$
p^{2}=4 \beta^{2} / \alpha^{4}=\gamma \delta R^{4} / b .
$$

In what circumstances, if any, does a nonzero value for at least one of the constants $A_{m}$ provide a solution? Application of the four boundary conditions, Eqs. (24) and (25), provide a system of four linear equations with unknowns $\mathbf{A}=\left[A_{1}, A_{2}, A_{3}, A_{4}\right]$,

$$
\mathbf{M A}=0 .
$$

The entries of the $4 \times 4$ matrix $\mathbf{M}$ depend on the parameters $\alpha$ (representing the length of the elastic line, Eq. (20)) and $p^{2}$ (ramp strength, Eq. (28)). The circumferential trajectory $\mathbf{A}=\mathbf{0}$ is a solution. The condition for stability of this equilibrium trajectory is its uniqueness, which requires nonsingularity of $\mathbf{M}$. We search for conditions yielding $\operatorname{det}(\mathbf{M})=0$, as then the circumferential trajectory will become unstable toward trajectories with an axial component.

a. The weak ramp, $p^{2}<1$. In this case the characteristic roots are imaginary, $a_{m}= \pm i \zeta_{1}, \pm i \zeta_{2}$, where $\zeta_{1}$ and $\zeta_{2}$ represent the two values of the expression,

$$
(\alpha / \sqrt{2}) \sqrt{1 \pm \sqrt{1-p^{2}}}
$$


The general solution may be written in terms of sines and cosines,

$$
\begin{aligned}
y(\sigma)= & A \cos \left(\zeta_{1} \sigma\right)+B \sin \left(\zeta_{1} \sigma\right) \\
& +C \cos \left(\zeta_{2} \sigma\right)+D \sin \left(\zeta_{2} \sigma\right) .
\end{aligned}
$$

For the ramp-free case, $p^{2}=0$, the corresponding matrix $\mathbf{M}$ is nonsingular for $\alpha<\pi / 2$, but its determinant vanishes at the critical value $\alpha=\pi / 2$. (In practice, we verified this statement by solving the boundary conditions for $B, C$, and $D$ in terms of $A$.) Therefore an elastic line of critical length $l=\pi R / 2^{3 / 2}$, lying along the base circle, is in a state of neutral equilibrium, a continuum of near-lying equilibrium trajectories with axial components emerging at this length. We know that sufficiently short elastic lines are stable along the base circle. So elastic lines with lengths less than the critical value are in stable equilibrium along the circle, but longer lines are unstable in the circumferential trajectory. The stable trajectory for lengths not much in excess of critical has pitch $\theta$ increasing like $\sin [(\pi / 2) \sigma]$. These results for the ramp-free case coincide with ones obtained previously [2].

For a very weak ramp, $p^{2} \ll 1$, we anticipate an increased critical value of the form $\alpha=(\pi / 2)+a p^{2}$, with a positive constant $a$. We find, to leading terms,

$$
\begin{aligned}
& \zeta_{1}=\left(\alpha^{2} / \pi\right) p \\
& \zeta_{2}=(\pi / 2)+\left(\alpha^{4} / 4\right)\left[a-\left(4 / \pi^{3}\right)\right] p^{2} .
\end{aligned}
$$

$\operatorname{Det}(\mathbf{M})$ becomes zero for a smallest value of $\alpha$ given by

$$
\alpha=(\pi / 2)+\left(\alpha^{4} / \pi^{3}\right)\left[1-\left(8 / \pi^{2}\right)\right] p^{2} .
$$

Iterating on the second (small) term, we get a critical length for instability,

$$
l / R=\left(\pi / 2^{3 / 2}\right)+\pi p^{2}\left[1-\left(8 / \pi^{2}\right)\right] / 16 \sqrt{2} .
$$

The construction of a ramp along the base circle stabilizes the elastic line on it beyond the ramp-free critical length $\pi R / 2^{3 / 2}$.

At the limit for a weak ramp, $p^{2}=1$, nothing unusual seems to happen. The characteristic roots are doubly degenerate, $\pm i \alpha / \sqrt{2}$, the general solution $y(\sigma)$ may be written in standard form, and we find the emergence of nonvanishing solutions at a value of $\alpha$ that solves the equation

$$
\alpha^{2}-2 \sin ^{2}(\alpha / \sqrt{2})-8 \cos ^{2}(\alpha / \sqrt{2})=0 .
$$

Numerical analysis discloses a root at about 1.6824, not much larger than the rampfree critical value $\alpha=\pi / 2 \approx 1.5708$. A weak ramp stabilizes the circumferential trajectory, but not by much.

b. The strong ramp, $p^{2}>1$. The characteristic roots are complex, consisting of the four combinations of the expression $\pm \zeta \pm i \eta$, where

$$
\zeta=\alpha \sqrt{p / 2} \cos (\tau / 2), \quad \eta=\alpha \sqrt{p / 2} \sin (\tau / 2)
$$

with

$$
\tau=\arctan \sqrt{p^{2}-1}
$$


The general solution $y(\sigma)$ is,

$$
\begin{aligned}
y= & A e^{\zeta \sigma} \cos (\eta \sigma)+B e^{\zeta \sigma} \sin (\eta \sigma) \\
& +C e^{-\zeta \sigma} \cos (\eta \sigma)+D e^{-\zeta \sigma} \sin (\eta \sigma) .
\end{aligned}
$$

We look first at the very strong ramp, $p^{2} \gg 1$. For sufficiently large $p^{2}$, it is a straightforward matter to show that $\operatorname{det}(\mathbf{M})$ does not vanish for any $\alpha>0$. (In practice, we solved the boundary conditions for $C$ and $D$ in terms of $A$ and $B$, then worked with the $2 \times 2$ matrix of coefficients that determine $A$ and $B$.) The solution $A=B=C=D=0$ is therefore unique. An arbitrarily long elastic line, started in the direction of the base circle, continues to wind around the circle in a stable trajectory, if the ramp is strong enough. (Recall from the Introduction and from [2] that the stable trajectory of a long elastic line unaided by a ramp becomes almost entirely axial in character even if its starting direction is circumferential.)

It follows that there must be a definite upper bound to the range of ramp strengths $p^{2}$ for which $\operatorname{det}(\mathbf{M})$ vanishes for some $\alpha>0$. Below this bound, circumferential elastic lines of length greater than that corresponding to the root $\alpha$ are unstable. For greater ramp strengths arbitrarily long elastic lines are stable along the base circle. (The alternative behavior, which does not occur, would be the existence of a critical root $\alpha\left(p^{2}\right)$ which gradually and indefinitely increases with increasing values of the argument, i.e., stability for longer and longer elastic lines with stronger and stronger ramps, or, still again, simple extension of the behavior found up to the limit of weak ramps.)

A numerical investigation of $\operatorname{det}(\mathbf{M})$ reveals that for $p^{2}$ about equal to 5.1738 , there is a smallest positive root of $\operatorname{det}(\mathbf{M})=0$ about equal to $2.0051 \quad(l / R \approx 1.4178)$. For greater values of $p^{2}$, there is no root, and $\mathbf{M}$ is nonsingular. Thus a ramp of strength $p^{2}=5.1738$ stabilizes the circumferential trajectory for elastic lines of lengths up to, but not beyond, $1.4178 R$. This value is not much greater than the ramp-free critical length $\pi R / 2^{3 / 2} \approx 1.1107 R$. Stronger ramps, even barely stronger ramps, stabilize the circumferential trajectory for an elastic line of any length, no matter how great.

c. Remark. We have a strong check on our program to determine the critical value of $\alpha$ for given ramp strength $p^{2}$ in the strong-ramp case $p^{2}>1$. Noting that it produced a value for $\alpha$ about equal to 1.5708 , close to $\pi / 2$, for $p^{2}=4$, we determined by an exact calculation that $\alpha=\pi / 2$ does indeed mark the singularity of $\mathbf{M}$ when $p^{2}=4$.

6. Possible biological implications. In the cell nucleus the primary "packaging unit" of a chromosome, the so-called nucleosome, consists of DNA wound in two complete turns on a cylinder-shaped protein core [4]. The cylinder is squat, about half as high as its diameter. The DNA is thought to be held on the cylindrical surface largely by means of attractive forces between negative ionic groups on the DNA and positive groups on the protein. In the following discussion we ignore the shallow pitch of the DNA that prevents self-intersection of its two turns and thus regard the DNA in a purely circumferential trajectory around the protein cylinder. 
We have seen that there is a tendency for DNA, as an elastic line, to straighten axially, along the generators of the cylinder. In the ramp-free case the critical length for stability as a circular arc, $l=1.1107 R$, is only about a sixth of a turn. Two circular turns of DNA (which define a DNA segment about equal to a persistence length, hence one that possesses significant bending resiliency) would be highly unstable toward a trajectory with a marked axial component. Thus there probably exists a "ramp" for the DNA, that is, a potential energy trough running along the circumference of the cylinder.

One interpretation of the ramp is physically obvious. If the DNA were to straighten axially, it would run off the ends of the squat cylinder, thereby losing its favorable energy of ionic attraction to the protein. Another possibility follows from the "oligomeric" nature of the protein core, which actually consists of an association of eight protein monomers. Perhaps the squat cylinder can be regarded as a folded telescope, which can elongate if the monomers dissociate, but only at the cost of a portion of the octameric association energy (i.e., attractive forces among the eight protein monomers that hold them together). The DNA can straighten axially as the protein core telescopes outward, but it is resisted in so doing by a ramp energy generated by the forces of attraction between segments of the telescope.

We saw that a subcritical ramp strength (below $p^{2}$ about 5.1738) can at best stabilize a circumferential trajectory of length $l=1.4178 R$, or only about a quarter of a turn. The two turns of nucleosomal DNA would appear to require a ramp strength above critical, for such ramp forces, no matter how slightly in excess of critical, can stabilize arbitrarily many circular turns. Suppose that the nucleosomal ramp in the "resting state" is slightly above the critical strength. Then the two DNA turns are stable. Now suppose a cellular modification reduces the ramp to a strength just below critical. Only a quarter of a turn would be stable. The two turns become markedly unstable, and the DNA extends axially, either off the ends of the protein core, or accompanied by the disruption of the protein core octamer. In either case a "regulatory" mechanism, exposing the DNA to metabolic activity, has operated.

We have an expression for the critical ramp strength, $p^{2}=5.1738$, with Eq. (28) for $p^{2}$ in terms of structural parameters. Plausible numerical modeling suggests that the nucleosomal ramp, interpreted as a resistance to dissociation of the octamer, may be near the critical value. These matters will be discussed in more detail elsewhere.

\section{REFERENCES}

[1] G. S. Manning, Relaxed elastic line on a curved surface, Quart. Appl. Math. 45, 515-527 (1987)

[2] G. S. Manning, The winding of a relaxed elastic line on a cylinder. Quart. Appl. Math. 45, 809-815 (1987)

[3] H. K. Nickerson and G. S. Manning, Intrinsic equations for a relaxed elastic line on an oriented surface, Geom. Dedicat. 27, 127-136 (1988)

[4] J. Darnell, H. Lodish, and D. Baltimore, Molecular Cell Biology, Scientific American Books, New York, 1986

[5] G. S. Manning, Elastic line deformed on a surface by an external field: intrinsic formulation and preliminary application to nucleosome energetics, Phys. Rev. A 38, 3073-3081 (1988) 